\title{
Exploring the micromechanical behaviour of sand-rubber mixtures using X-ray micro-tomography
}

\author{
Zhuang Cheng ${ }^{1 *}$, Jianfeng Wang ${ }^{1}$, and Wei $\mathrm{Li}^{2}$ \\ ${ }^{1}$ Department of Architecture and Civil Engineering, City University of Hong Kong, 999077, Hong Kong, China \\ ${ }^{2}$ Faculty of Engineering, China University of Geosciences, 430074, Wuhan, China
}

\begin{abstract}
The micromechanical behaviour of sand-rubber mixtures (SRMs) under monotonic triaxial shear were investigated using X-ray micro-tomography. The localisation of sand particle rotations that occurred in a pure sand sample under shear was inhibited in the sand mixed with $30 \%$ rubber grains by mass. Meanwhile, the SRMs exhibited an evolution of sand-sand contact coordination number that is not negatively correlated with sample porosity, dramatically different from that was observed in pure sands. Substantially increasing anisotropy degree of sand-rubber contacts compared with minor changes of sandsand contact fabric was observed, implying the increasingly important role of sand-rubber contacts in the transmission of deviatoric loads as the shear of SRMs progressed.
\end{abstract}

\section{Introduction}

In recent years, accelerating increase of waste rubber tyres as a result of rapid rising vehicles has posed a great threat to the environment. Reusing them as construction materials in a wide range of civil engineering applications including slope engineering, embankments, seismic isolation systems and drainage layers, etc. has become a promising solution to this environmental issue $[1,2]$.

Waste rubber grains are usually mixed with sands to form sand-rubber mixtures (SRMs), which have various technical advantages over pure sands in engineering applications. For instance, SRMs are lighter in weight and generally have higher seismic resistance and hydraulic conductivity than pure sands. Composed of two types of grains (i.e., sand grains and rubber grains) with substantially different stiffnesses, SRMs generally have more complex mechanical behaviour than pure sands. The complexity stems from the existence of multiple load-transferring mechanisms including loads transferring through sand-sand contacts, sand-rubber contacts and rubber-rubber contacts within SRMs [3-6]. This intrinsic feature of SRMs has dominated the fact that their mechanical behaviour is affected by various factors including rubber content, rubber shape and size as well as soil types. While the mechanical behaviour of SRMs have been intensively studied in recent years, the underlying particle-level mechanisms remain poorly understood because of the lack of experimental tools to investigate the grain-scale behaviour of SRMs.

Recently, the rapid development of X-ray imaging techniques has allowed the visualization of grain-scale mechanical behaviour of granular materials at spatialresolution up to microns [7-11], providing new possibilities to the grain-scale investigation of SRMs. This paper presents the use of X-ray micro-tomography to explore the micromechanical behaviour of SRMs under monotonic triaxial compression, with a focus on the fabric evolution of sand-sand contacts and sandrubber contacts as well as grain kinematics.

\section{Experiments and data analysis}

\subsection{Experimental setup, test materials, and data acquisition}

The experiments were performed using a specially fabricated miniature loading apparatus [12] in conjunction with an X-ray micro-tomography scanner at the Shanghai Synchrotron Radiation Facility Centre (SSRF). The X-ray setup produced X-ray CT images with a spatial resolution of $6.5 \mu \mathrm{m}$ and allowed for the acquisition of full-field scans of the tested sample $(8 \mathrm{~mm}$ $\times 16 \mathrm{~mm}($ Diameter $\times$ Height $))$ at different loading stages of a test.

To perform the test, two SRM samples (i.e., a Leighton buzzard sand-rubber mixture sample (LBS-R) and a glass bead-rubber mixture sample (GB-R)) with a rubber content of $30 \%$ by mass (equivalent to $50 \%$ by volume) were produced using LBS $(0.4-0.8 \mathrm{~mm})$, GB $(0.3-0.6 \mathrm{~mm})$ and rubber grains $(0.3-0.6 \mathrm{~mm})$. The materials have distinctively different stiffness: LBS, GB and rubber have a Young's modulus of $52 \mathrm{MPa}, 58 \mathrm{MPa}$ and $1.02 \mathrm{kPa}$ respectively. LBS and GB have a Poisson's ratio of 0.25 and 0.30 , respectively, while the rubber's Poisson's ratio is close to 0.50 . The samples were dry and were prepared by air pluviation. To avoid segregation, the sand grains and rubber grains were

\footnotetext{
* Corresponding author: zhuacheng2-c@my.cityu.edu.hk

A video is available at https://doi.org/10.48448/dhvs-1868
} 
pluviated into the sample in a batchwise and alternating manner, i.e., tens of sand grains followed by tens of rubber grains were repeatedly pluviated into the sample until it was fully filled. These samples, together with an LBS sample and a GB sample (with similar grain sizes), were triaxially compressed under a confining stress of $500 \mathrm{kPa}$ within the miniature loading apparatus, respectively $[8,13]$. Each test was paused at the specific axial strain levels for the acquisition of CT images of the sample. Table 1 summaries the sample initial properties and the axial strain levels for CT scanning.

Fig. 1 presents the stress-strain curves of the samples during shear, on which the locations of CT scanning are marked with red circles. The sand samples exhibited a clear peak stress ratio with volumetric dilation occurring during the post-peak of shear (i.e., 4.94-15.34\% for LBS and $3.96-12.14 \%$ for GB), while the SRMs showed increasing stress ratio with volumetric compression throughout the tests.

Table 1. Summary of test conditions of the samples

\begin{tabular}{|c|c|c|}
\hline Sample & $\begin{array}{c}\text { Pre-shear } \\
\text { porosity }\end{array}$ & Scan acquired at axial strain/\% \\
\hline GB & 0.331 & $0,2.02,3.96,8.06,12.14$ \\
\hline GB-R & 0.167 & $\begin{array}{c}0,2.67,3.85,5.77,7.68,9.66, \\
11.81,13.5,15.53,17.49,18.92\end{array}$ \\
\hline LBS & 0.343 & $0,0.98,4.94,104,15.34$ \\
\hline LBS-R & 0.182 & $\begin{array}{c}0,1.26,2.27,4.19,5.98,7.84, \\
9.8,11.52,13.4,15.39\end{array}$ \\
\hline
\end{tabular}

(a)

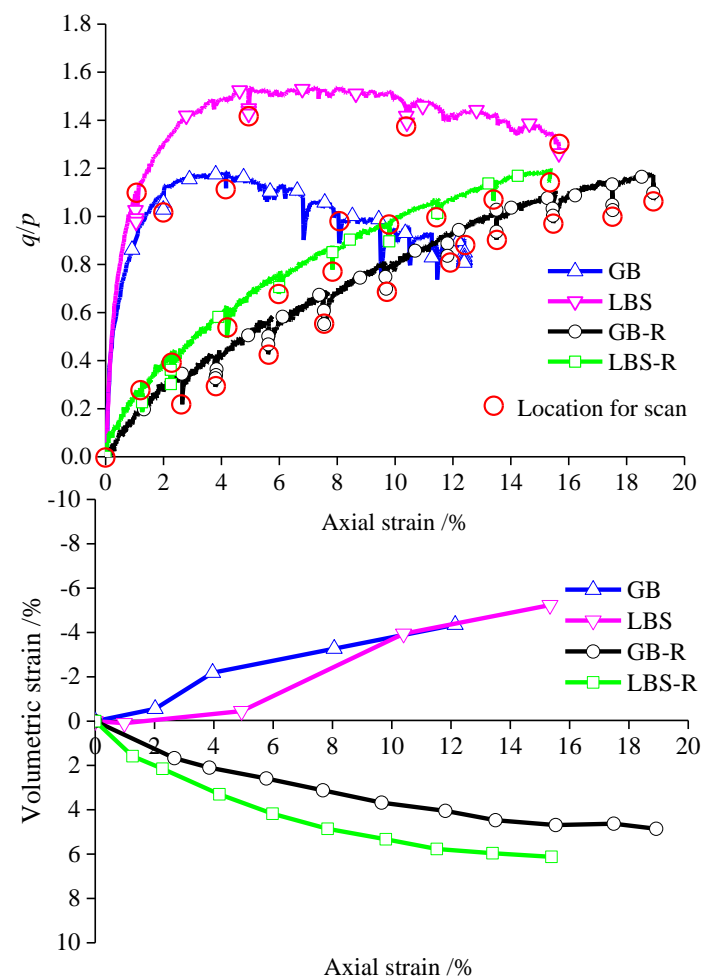

Fig. 1 Stress-strain curves: (a) stress ratio v.s. axial strain; (b) volumetric strain v.s. axial strain

\subsection{Image processing and analysis}

\subsubsection{Image segmentation and particle separation}

Image processing and analysis was performed on the acquired CT images of the samples following the methods presented in [13]. Specifically, each original CT image was first filtered using a low-pass filter [14] to remove the noises. Then, global thresholding and an improved region growing method [15] were applied to identify the different phases of the sand samples (i.e., the sand and void phases) and the SRM samples (i.e., the sand, void and rubber phases), respectively. From the segmented CT image, the sand phase was extracted and stored in a binary image. Subsequently, a marker-based watershed algorithm [16] was applied to the binary image to determine a set of watershed lines, which was used to separate individual sand grains. Based on the image of separated sand grains, the grain geometrical information including grain volume, surface area and centroid coordinate were quantified using an intrinsic MATLAB function 'regionprops'.

\subsubsection{Identification of grain contacts}

The interparticle contact regions between any two contacting sand grains were extracted based on a logical 'AND' operation between the binary image of sand phase and the binary image of watershed lines that were determined in the watershed segmentation [8]. To assign the isolated contact regions to the contacting sand particle pairs, a morphological dilation of the image of contact regions was implemented. A contact region was assigned to a particle if at least one voxel of the increased region of that contact region belongs to the particle region.

To determine the contact regions of any sand-rubber contact (i.e., the contact between a sand and a rubber grain), a morphological dilation of the binary image of sand phase was implemented. The sand-rubber contact regions were determined as the intersected regions between the dilated sand phase and the rubber phase. The assignment of sand-rubber contact regions to a sand grain followed the same procedure to the sand-sand contacts.

\subsubsection{Quantification of grain kinematics}

The kinematics of sand particles including particle displacements and particle rotations during each load increment were acquired based on a particle tracking method [11]. The method assumed the sand grains experience little crushing and used either grain volume or grain surface area as the particle tracking criterion to track the sand grains. In the method, the displacement and rotation of a grain were calculated as the centroid coordinate difference and the orientation difference of the grain between the start and the end loading states, respectively. 


\section{Experimental results}

\subsection{Particle kinematics}

Fig. 2 presents 3D maps of the sand particle displacements and rotations of LBS-R and LBS during the last shear increment (i.e., axial strain of 13.4-15.39\% for LBS-R and $10.4-15.34 \%$ for LBS). Note that the kinematics were measured with respect to the previous CT snapshot. Only the results of a single shear increment of the two samples are presented here. Readers are referred to the study [13] for a more detailed presentation. LBS-R and LBS revealed a similar pattern of particle displacement, as depicted in Figs. 2a and 2c. LBS-R exhibited a disorganised distribution of low particle rotations (usually smaller than $20^{\circ}$ ) during its last shear increment (Fig. 2b). In contrast, LBS revealed a clear localised band in the particle rotation map (Fig. $2 d)$. Compared to LBS-R, LBS revealed a higher degree of particle rotations within the shear band. This is attributed to the different interparticle contact types between the two samples. In LBS, all external loads were transferred through sand-sand contacts, which had rather small contact areas. In contrast, the sand-rubber contacts also participated in the load transmission of LBS-R. These sand-rubber contacts had large contact areas. The larger contact areas of sand-rubber contacts than sand-sand contacts led to a higher anti-rotation resistance of the LBS grains in SRM than in pure sands. Therefore, LBS-R had lower rotations of LBS grains than the LBS sample.

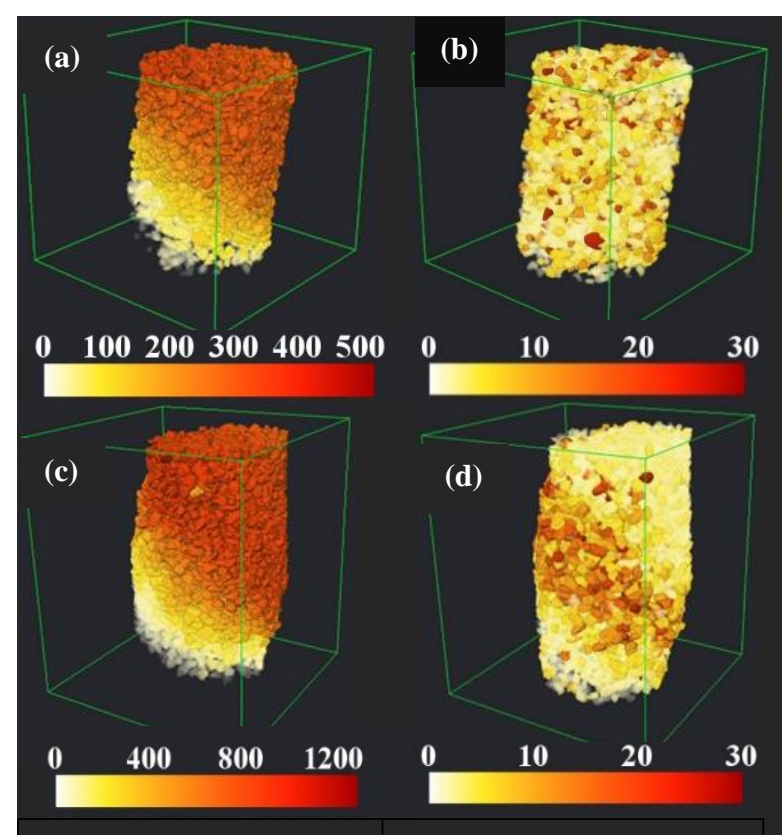

Particle displacement/ $\mu m$ Particle rotation/degrees

Fig.2 Displacements (a) and rotations (b) of sand grains in LBS-R during the last shear increment; displacements (c) and rotations (d) of sand grains in LBS during the last shear increment

\subsection{Contact fabric}

Fig. 3 depicts the evolution of average coordination number $(\mathrm{CN})$ of sand-sand contacts within the samples. It should be mentioned that the isolated contacting regions of any contacting particle pair were regarded as a single contact. As seen in Fig. 3a, both GB-R and LBS$\mathrm{R}$ experienced three stages of average $\mathrm{CN}$ change, i.e., a gradual increase of average $\mathrm{CN}$ at the initial shear stage, followed by a relatively stable fluctuation of the values and ended with a gradual decrease of average CN. As stated above, both samples experienced volumetric compression throughout the entire test. The phenomena implied that the average $\mathrm{CN}$ does not have a negative correlation with sample porosity. These were dramatically different from those observed in the pure sand samples: the average CN of GB and LBS decreased throughout the shear progress where volumetric dilation occurred (i.e., at the axial strain of 3.96-12.14\% for GB and $4.94-15.34 \%$ for LBS, respectively, see Fig. 3b). This was attributed to the different interparticle contact interaction modes between pure sands and SRMs [13].

(a)

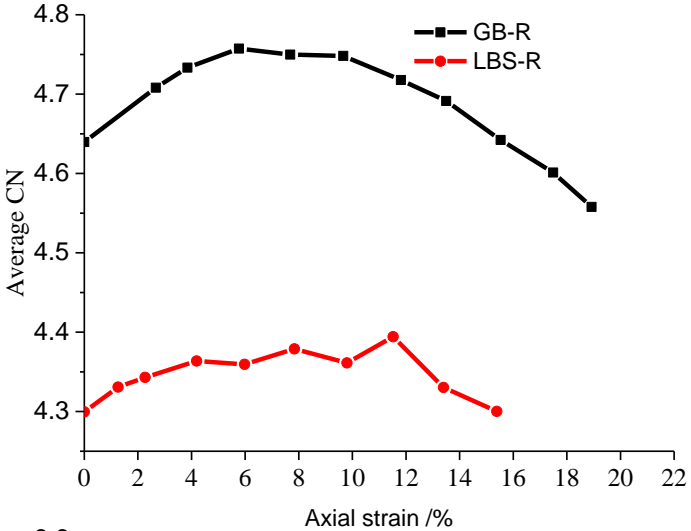

(b)

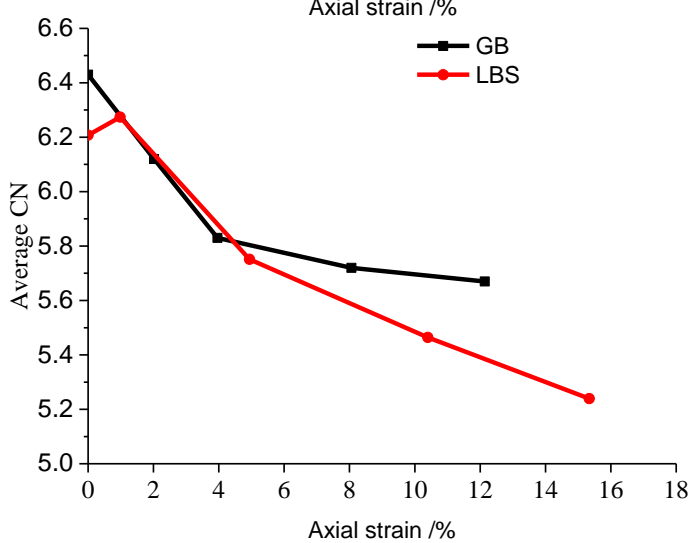

Fig. 3 Sand-sand contact coordination number evolution: (a) in GB-R and LBS-R samples; (b) in GB and LBS samples

To quantify the fabric anisotropy of sand-sand contacts and sand-rubber contacts, anisotropy degree $a$ of sand-sand contacts and sand-rubber contacts were calculated based on the orientations of sand-sand branch vectors and sand-rubber branch vectors, respectively [13]. Specifically, the sand-sand branch fabric tensor was calculated following [17]. For the sand-rubber contacts, a fabric tensor $F_{i j}$ was defined according to Eq. (1):

$$
F_{i j}=\frac{1}{\sum \omega_{k}} \sum_{k=1}^{N} \omega_{k} n_{i}^{k} n_{j}^{k},
$$


where $N$ is the number of sand-rubber contacts, $\omega_{k}$ and $n_{i}^{k}$ are the area weight, and the component of the $k^{\text {th }}$ unit orientation vector along direction $i$, respectively. The anisotropy degree $a$ of the sand-sand fabric tensor and sand-rubber fabric tensor was then determined according to [18]. Fig. 4a presents the variation of anisotropy degree $a$ of sand-sand contacts with the axial strain for the four samples. Both pure sand samples experienced a gradual increase followed by a relatively stable stage of $a$, while there were only minor changes of $a$ throughout the shear of SRMs. Fig. $4 \mathrm{~b}$ depicts the evolution of $a$ of sand-rubber contacts of the SRMs. Unlike the sand-sand contacts, the sand-rubber contacts of the SRMs exhibited a substantial increase during the shear, indicating increasing contact forces of sandrubber contacts along the deviatoric loads. The phenomenon implied that sand-rubber contacts played an important role in the transmission of deviatoric loads during the shear of SRMs.

(a)

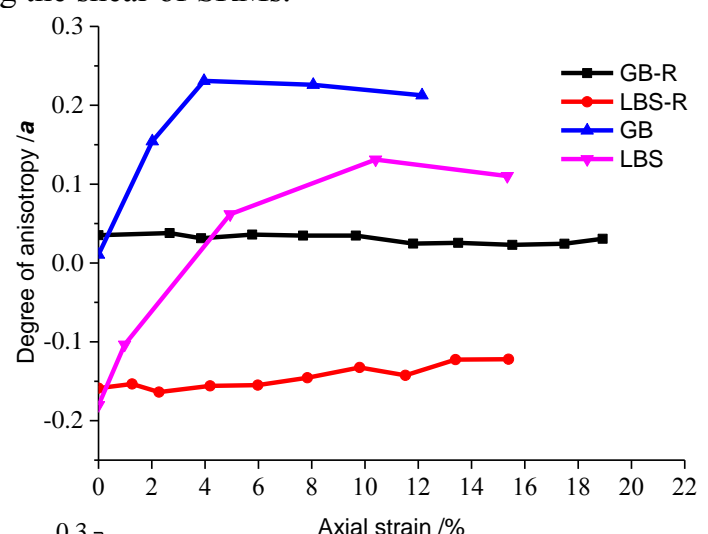

(b)

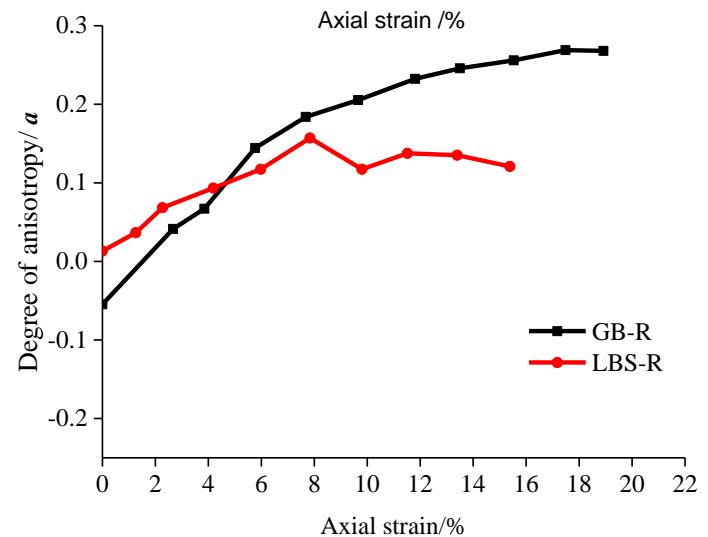

Fig.4 Anisotropy degree $a$ of (a) sand-sand contacts and (b) sand-rubber contacts

\section{Conclusions}

Triaxial compression tests were performed on pure sands and SRMs with a rubber content of $30 \%$ by mass. The grain-scale behaviour was investigated based on Xray $\mathrm{CT}$ images of the samples during the shear. It was observed that the localisation of sand particle rotations that occurred in a pure sand sample did not appear in the corresponding SRM sample. This was related to the stronger anti-rotation effects of sand-rubber contacts on the sand grains than sand-sand contacts, which resulted from the larger contact areas of the former in the SRM than the latter in the pure sand sample.
Besides, the average $\mathrm{CN}$ of sand-sand contacts of SRMs experienced an initial increase, followed by a small fluctuation and a gradual decrease during the shear. Unlike the pure sands, the average CN of SRMs did not have a negative correlation with the varying sample porosity. Meanwhile, increasing anisotropy degree of sand-rubber contacts compared with minor changes of sand-sand contact fabric was observed throughout the test, suggesting the increasingly important role of sandrubber contacts in the transmission of deviatoric loads as the shear of SRMs progressed.

This study was supported by the General Research Fund No. CityU 11213517 from the Research Grant Council of the Hong Kong SAR, Research Grant No. 51779213 from the National Science Foundation of China, and the BL13W beamline of the Shanghai Synchrotron Radiation Facility (SSRF).

\section{References}

[1] P.S.H. Poh, B.B. Broms, J. Perform, 9, 76-79 (1995)

[2] H. Tsang, S.H. Lo, X. Xu, M.N. Sheikh, Earthq. Eng. Struct. Dyn, 41, 2009-2024 (2012).

[3] J. Zhang, X. Chen, J. Zhang, P. Jitsangiam, X. Wang, Particuology, (2020, In Press).

[4] J.C. Lopera Perez, C.Y. Kwok, K. Senetakis, Geotext. Geomembranes, 45, 81-97 (2017).

[5] L. Gong, L. Nie, Y. Xu, H. Wang, T. Zhang, C. Du, Y. Wang, Constr. Build. Mater, 205, 574-585 (2019)

[6] K. Taghizadeh, H. Steeb, V. Magnanimo, S. Luding, Elastic waves in particulate glass-rubber mixture: experimental and numerical investigations/studies, in: Powders and Grains, Montpellier, France, 2017

[7] E. Andò, S.A. Hall, G. Viggiani, J. Desrues, P. Bésuelle, Acta Geotech, 7, 1-13 (2012)

[8] Z. Cheng, J. Wang, Soils Found, 58, 1492-1510 (2018)

[9] Y. Higo, F. Oka, S. Kimoto, T. Sanagawa, Y. Matsushima, Soils Found, 51, 95-111 (2011)

[10] K.A. Alshibli, M.F. Jarrar, A.M. Druckrey, R.I. AlRaoush, JGGE, 143, 04016097, 2017

[11] Z. Cheng, J. Wang, Powder Technol, 328, 436-451 (2018)

[12] Z. Cheng, J. Wang, M.R. Coop, G. Ye, Front. Struct. Civ. Eng, 14, 357-373 (2020)

[13] Z. Cheng, J. Wang, W. Li, Soils Found, 60, 12511268 (2020)

[14] P. Perona, J. Malik, IEEE Trans. Pattern Anal. Mach. Intell, 12, 629-639 (1990)

[15] Z. Cheng, J. Wang, Powder Technol, 368, 80-89 (2020)

[16] C. Wählby, I.-M. Sintorn, F. Erlandsson, G. Borgefors, E. Bengtsson, Combining intensity, J. Microsc, 215, 67-76 (2004)

[17] M. Satake, Fabric tensor in granular materials, in: Deform. Fail. Granul. Mater., Balkema, Amsterdam, 1982

[18] S. Yimsiri, K. Soga, Géotechnique, 60, 483-495 (2010) 